\title{
Cepheids as tracers of star formation in M $31^{\star, \star \star}$
}

\section{Observations and identifications}

\author{
E.A. Magnier ${ }^{1,2,3,4,5}$, T. Augusteijn ${ }^{1,2,6}$, S. Prins ${ }^{1,2}$, J. van Paradijs ${ }^{1,2,7}$, and W.H.G. Lewin ${ }^{5}$ \\ 1 Astronomical Institute "Anton Pannekoek", Kruislaan 403, 1098 SJ Amsterdam, The Netherlands \\ 2 Center for High Energy Astrophysics, Kruislaan 403, 1098 SJ Amsterdam, The Netherlands \\ 3 University of Illinois, Astronomy Dept. Bldg. 103, 1002 W. Green St., Urbana, IL 61801, U.S.A. \\ 4 Astronomy Dept. 351580, University of Washington, Seattle, WA 98195, U.S.A. \\ 5 Massachusetts Institute of Technology, Room 37-624, Cambridge, MA 02139, U.S.A. \\ ${ }^{6}$ European Southern Observatory, Casilla 19001, Santiago 19, Chile \\ 7 Physics Department, University of Alabama in Huntsville, Huntsville, AL 35899, U.S.A.
}

Received November 14, 1996; accepted April 2, 1997

\begin{abstract}
Cepheid variables can be used for more than just a distance indicator. Since the age can be determined from the period, they can be used to trace the star formation history of a galaxy. We have identified 130 new Cepheid variable candidates in M 31, particularly along the spiral arm regions in the north-east of the galaxy. These areas had not been searched for Cepheids in the previous study by Baade and collaborators. The contamination of our sample by other types of variables is low $(<3 \%)$. For $V<21$, the completeness is $\sim 53 \%$. These Cepheids will be used to explore the star formation history in M 31 .
\end{abstract}

Key words: (stars: variables:) Cepheids — stars: formation - galaxies: individual: M 31 - galaxies: stellar content - (cosmology:) distance scale

\section{Introduction}

Cepheid variables are well known for their use as a reliable distance indicator. M 31 was among the first galaxies to have the distance measured, in part using Cepheid variables identified by Hubble (1929). A later, more extensive search for Cepheid variables was performed by Baade \&

Send offprint requests to: E.A. Magnier: gene@astro.uva.nl * Based on observations made with the Isaac Newton Telescope operated on the island of La Palma by the Royal Greenwich Observatory in the Spanish Observatorio del Roque de los Muchachos of the Instituto de Astrofisica de Canarias.

** Table 1 and Figures 5 to 10 are only available in electronic form at CDS via anonymous ftp to cdsarc.u-strasbg.fr (130.79.128.5) or vai http://cdsweb.u-strasbg.f/Abstract.html
Swope $(1962,1964)$ and Gaposhkin (1963). Since then, substantial work has gone into improving the determinations of the periods and apparent magnitudes of these Cepheids, and in making corrections for the effects of extinction and metallicity variations (e.g., Welch et al. 1986; Freedman \& Madore 1990). The result of this work has been a reliable determination of the distance to M 31 with a remaining uncertainty of only $10 \%$, and possibly as little as $5 \%$.

Another important use of Cepheids is due to the fact that their ages can be determined from the pulsation period. The Cepheid variability occurs when the star passes through the instability strip, after the star has evolved away from the main sequence. The mass of the star determines the period of the variability, and the mass is also directly related to the main-sequence lifetime of the star. Therefore, the age of the star can be determined from the period of the oscillation. This relationship was first discussed in the 1960s (Young 1961; Efremov \& Kopylov 1967), and has since been confirmed observationally (see Tsvetkov 1988 for an overview). The typical range of Cepheid periods observed in the Local Group galaxies, between $\sim 1$ and 50 days, corresponds to ages of $\sim 250$ Myrs and 25 Myrs, respectively. Thus, Cepheid variables can be used to trace stellar populations with intermediate ages.

Substantial work has already been done to identify Cepheids in M 31 (Baade \& Swope 1962, 1964; Gaposhkin 1963). These searches were limited to a small portion of M 31 (see Fig. 1) and ignore the spiral arm regions, where star formation is most active. As part of an unrelated variability study in M 31, we have surveyed a large portion of the eastern and southern spiral arm regions. We have used this dataset to identify Cepheids. In this article, we report on the analysis of the data and identification of the 


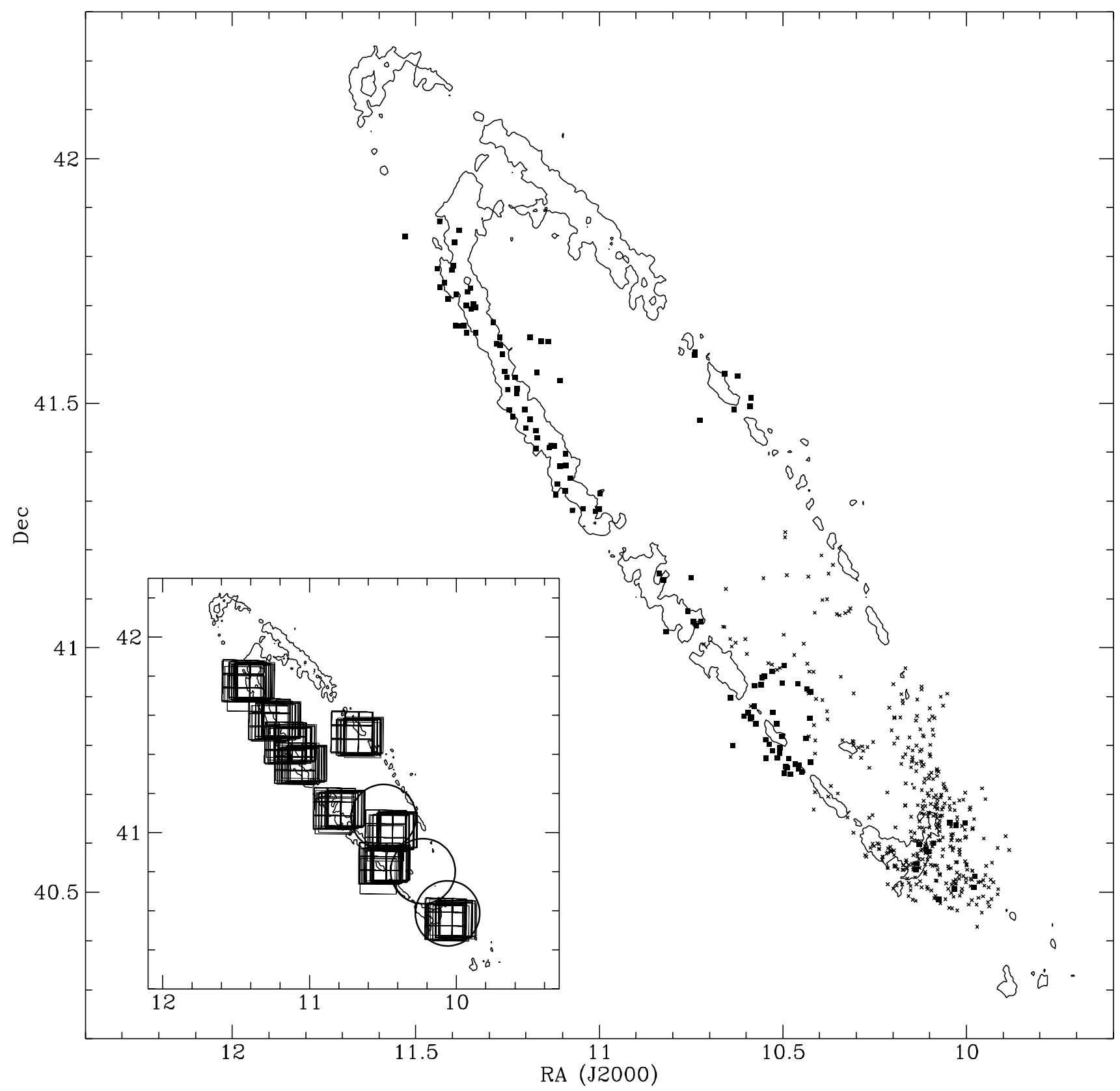

Fig. 1. This figure shows the locations of the Cepheids identified by previous researchers (Baade \& Swope 1962, 1964; Gaposhkin $1963-\times$ 's) and our new Cepheid candidates (squares) overlayed on an Hi contour map (Unwin 1980). The inset shows the locations of our 9 fields (squares) and the Baade's Fields I - III. Baade's field IV is beyond the southern edge of this figure

variables. In a companion paper, we will discuss the distribution of the Cepheids in terms of the star formation history.

\section{Observations}

We observed 9 fields in M 31 during a period of 25 days in the fall of 1993 (see Fig. 1). The observations were performed using both the $2.5 \mathrm{~m}$ Isaac Newton Telescope
(INT) at the Observatorio de Roque de los Muchachos, La Palma, and the $1.3 \mathrm{~m} \mathrm{McGraw-Hill} \mathrm{telescope} \mathrm{of} \mathrm{the}$ Michigan-Dartmouth-MIT (MDM) Observatory at Kitt Peak. The observations at the INT were performed between Sept. 9 and Sept. 17, while those at MDM were performed between Sept. 24 and Oct. 4, 1993.

The observations at the INT were performed using the Ford/Pennypacker $2048^{2}$ CCD and a Kitt Peak $V$ filter. For the $\mathrm{f} / 7.5$ focus of the INT, this CCD has a pixel scale 
of 0.367 arcseconds per pixel, giving the $2048^{2}$ CCD a field of view of 12.5 arcminutes. The observations at MDM were performed using the $2048^{2}$ Tektronics CCD known as "Wilbur" (Metzger et al. 1993) at the f/7.5 focus station of the $1.3 \mathrm{~m}$ telescope with a Kitt Peak $V$ filter. The CCD was read out with a $2 \times 2$ pixel binning factor, giving a pixel scale for this setup of 0.637 arcsec per pixel for a total field of view of roughly 10.9 arcminutes, similar to that of the INT observations.

The weather at both sites was good for most of the allotted period of time: Of the 20 total nights allocated to this project, four (Sept. 9, 17, 24, and 30) were lost to weather or equipment problems, several of the nights at both sites were photometric, and the rest of the nights had occasional minor clouds. A small amount of clouds was not a severe problem because differential photometry was used to determine the throughput for a given image. However, on the nights near full moon (Sept. 30), small amounts of cloud coverage increased the sky background drastically, and reduced the effective sensitivity. The measured seeing at the INT site ranged from 0.8 to 2.0 arcseconds, while that at the $1.3 \mathrm{~m}$ MDM site was in the range 1.2 to 2.2 arcseconds.

\section{Analysis}

Standard reduction consisting of bias subtraction and flat fielding was performed on the images using the ESOMidas package and our own software called "Mana" (Magnier 1996). The INT data required special care in the bias subtraction as there were significant variations in the bias across the chip. The images were corrected using a template of the bias plus an offset determined from the overscan for each image.

The INT images had substantial variations in the point spread function (PSF) as a function of position on the image, possibly due to a mis-alignment of the CCD in the optical plane. We split each of these images into 9 subimages to minimize this problem. This problem was less severe for the MDM data, so that the entire images could be kept intact, but variations in the PSF with position for both telescopes continued to plague the analysis of all images during relative photometry, and forced some variation in the standard analysis, as discussed below.

Photometry was performed with the program DoPHOT (Mateo \& Schechter 1989). This is a PSF fitting program which is designed to be easy to use automatically with a large number of images; DoPHOT needs a relatively small amount of information to start running, and it runs without any interaction with the user. A complete discussion of DoPHOT can be found in Mateo \& Schechter (1989). Freedman (1989) also discusses DoPHOT in comparison with other commonly used photometry routines.

DoPHOT models the objects on an image using an elliptical Gaussian with 7 free parameters: a zero level (sky), the central intensity $\left(I_{0}\right)$, the centroid $(X$ and $Y$ ), the semi-major and semi-minor axes of the ellipse $\left(\sigma_{x}\right.$ and $\left.\sigma_{y}\right)$, and the angle between the semi-major axis of the ellipse and the pixel coordinate system $(\alpha)$. For a particular image, DoPHOT uses a single set of values for the 3 "shape" parameters $\left(\sigma_{x}, \sigma_{y}\right.$, and $\left.\alpha\right)$ to represent the PSF. DoPHOT distinguishes between stars (which are well fit with the PSF) and extended objects (which are well fit by a profile significantly more extended than the PSF). DoPHOT reports instrumental magnitudes and positions in pixel coordinates for all objects as determined from these Gaussian fits.

Astrometry was performed on each image. Two databases were used as a reference. For most images, the MIT/Amsterdam CCD survey of M 31 (Magnier et al. 1992; Haiman et al. 1993) was used as an astrometric reference. For images without sufficient overlap with this survey, the survey by Berkhuijsen et al. (1988) was used, after correction for the systematic error reported in the astrometry (Magnier et al. 1993a). Linear astrometric conversions were used; i.e. translations, scaling, and rotations were included in the conversion between pixel coordinates $(X, Y)$ and sky coordinates $(\alpha, \delta)$. The astrometry for certain images was determined from other program observations of the same field, for which the above astrometric parameters had been succesfully determined. Typically between 30 and 100 stars from either of the reference catalogs were identified on each CCD frame. The residuals of the fit were also measured to provide an estimation of the astrometric error, which we determined to be typically $\sim 1.0$ arcseconds, dominated by the errors in the original catalogs.

\section{Relative photometry}

Relative photometry was performed on the images to convert the instrumental magnitudes to a common system. This allows us to make corrections for a variety of effects which may alter the throughput for a given image, in particular small amounts of clouds.

For every star on every image, there exists a relationship between the observed instrumental magnitude $m$,

$m=-2.5 \log \left(N_{\mathrm{el}}\right)+2.5 \log (t)$

and the apparent magnitude in a common system $M_{\mathrm{rel}}$ :

$m_{i, j}=M_{\mathrm{rel}, j}+M_{\mathrm{cal}, i}$.

The subscripts $i$ and $j$ refer to a particular image and a particular star, respectively. $M_{\text {cal }}$ is a correction for the throughput for a given image, and may incorporate effects such as cloud level. The goal of relative photometry is to determine $M_{\text {cal }}$ for each image, then use this $M_{\text {cal }}$ to find $M_{\text {rel }}$ for all stars from Eq. (2). Once one has $M_{\text {rel }}$ for each star, one can then convert them to a standard system, such as the Johnson system, using appropriate color corrections. This last step is in general more inaccurate 
for a variety of reasons, particularly because of the undersampling of existing photometry bands (see Young 1992). For the purpose of identifying variable stars, however, it is more important to have an accurate relative magnitude in an ill-defined system than well-calibrated magnitudes in a commonly used system.

We performed relative photometry according to the scheme outlined above, using an iterative method to minimize the $\chi^{2}$, defined as

$\chi^{2}=\sum_{i, j}\left(m_{i, j}-M_{\mathrm{rel}, j}-M_{\mathrm{cal}, i}\right)^{2} / \sigma_{i, j}^{2}$

where $\sigma_{i, j}$ is the error in the measurement $m_{i, j}$. For the present dataset, some modifications were necessary. First, we measured the $\chi^{2}$ for each star independently and removed those stars with unusually high $\chi^{2}$ values. This is needed to remove both the true variable stars from the calibration, as well as those stars which have a single or a few extreme outlying points, due to, e.g., cosmic ray hits or the star falling on a bad column. We also found that the residuals for a given image were a clear function of the position on the image. We traced this problem to the variation of the PSF across the images, for both telescopes. Since the model PSF is kept fixed for a given image (or portion of an image in the case of the INT images), stars which fit the model less well than other stars on the same image will have their flux poorly measured. Thus, a trend across the image in the size of the PSF is translated to a trend in the effective magnitude of a star measured at that position. To compensate for this effect, we modified Eq. (2) to incorporate a trend across the image:

$$
\begin{aligned}
m_{i, j}= & M_{\mathrm{rel}, j}+M_{\mathrm{cal}, i}+A_{i} x+B_{i} y+C_{i} x^{2} \\
& +D_{i} y^{2}+E_{i} x y+F_{i}
\end{aligned}
$$

where $A_{i}$ through $F_{i}$ are kept fixed for each image and $x$ and $y$ are the position of a measured star on an image (subscripts dropped for clarity). We solved the system for $A_{i}$ through $F_{i}$ along with the $M_{\text {rel }}$ and $M_{\text {cal }}$ terms. In fact, this last correction was not crucial; the actual magnitude of the correction introduced by the terms $A_{i}$ through $F_{i}$ was not very large compared to the variability of interest: typically only about $5-10 \%$. The fact that every frame had an arbitrary zero point allows a simple connection of the data from the INT $2.5 \mathrm{~m}$ and the data from the MDM $1.3 \mathrm{~m}$. In practice, we made initial guesses at the relative zero points of the solutions by correcting for the relative areas of the two telescopes. All data could then be processed simultaneously. The remaining scatter observed for non-variable stars which were bright enough $(V \lesssim 20)$ that photon noise was not significant was $1-2 \%$. The relative photometry was converted to the Johnson system by calibrating each field relative to the MIT/Amsterdam CCD survey of M 31 (Magnier et al. 1992; Haiman et al. 1993).

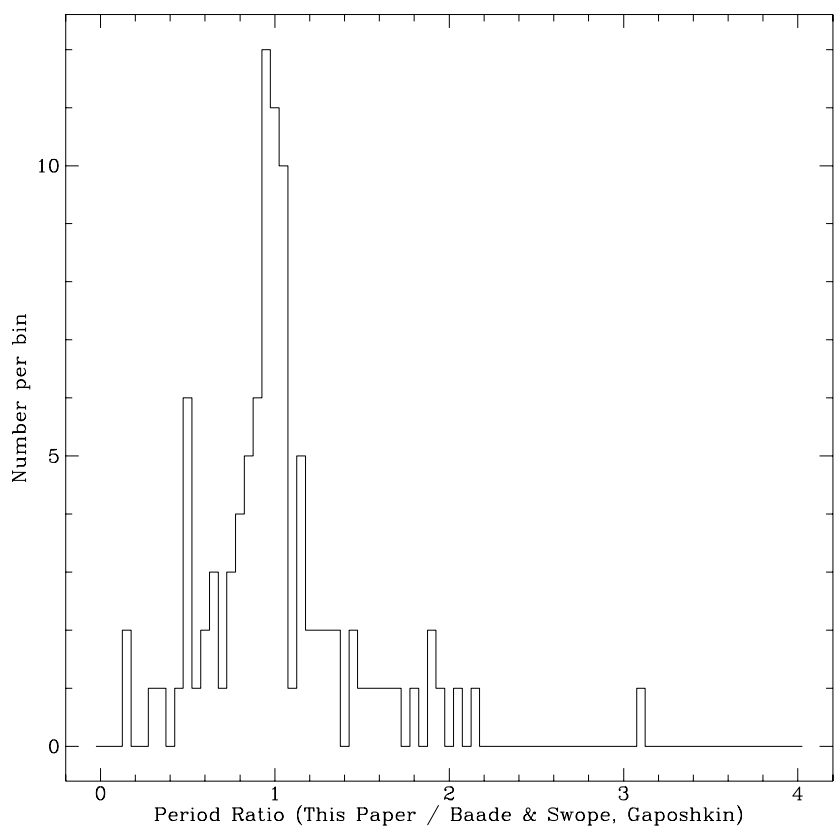

Fig. 2. Histogram of period ratios for M 31 Cepheids observed by us and by Baade \& Swope $(1962,1964)$ or Gaposhkin (1963)

\section{Results}

A total of 602 stars with $\chi^{2}$ larger than 10 was considered as possible candidates. The light curves of these stars were individually inspected and a variety of variable stars and possible variables was found. To identify the Cepheid variables in the sample, we searched for stars with light curves similar in appearance to known Galactic and LMC Cepheids. Of the 602 significantly variable stars, 228 were identified as Cepheid candidates. The remaining variable stars did not have light curves clearly similar to Cepheid light curves: e.g., short period variables with periods $<1$ day or erratic variables with only a small number of points far from the mean. Rough estimates of their periods were made by eye. We did not attempt to fit model light curves rigorously, partly because of the poor sampling, but also because exact periods are not crucial to our goals. We also determined the $V$ magnitude at maximum light, $V_{\max }$, for each candidate. The error on $V_{\max }$ varied considerably, from $\sim 0.02$ mag to 0.2 , depending on whether the peak of the light curve was observed or not.

Of the 228 Cepheid candidates, 97 were previously identified by Baade \& Swope $(1962,1964)$ and Gaposhkin (1963), particularly in our south-most field. Because they have a much larger amount of data covering a larger baseline, their period determinations are far more accurate than ours. Figure 2 shows a histogram of the ratio between their measurement of the periods and ours for each star found in common. The central peak has a $1 \sigma$ width of about $15 \%$. A peak can also be seen at 0.5 due to aliasing affecting our determination. Most of the error in our period determination is due to the poor sampling, since 


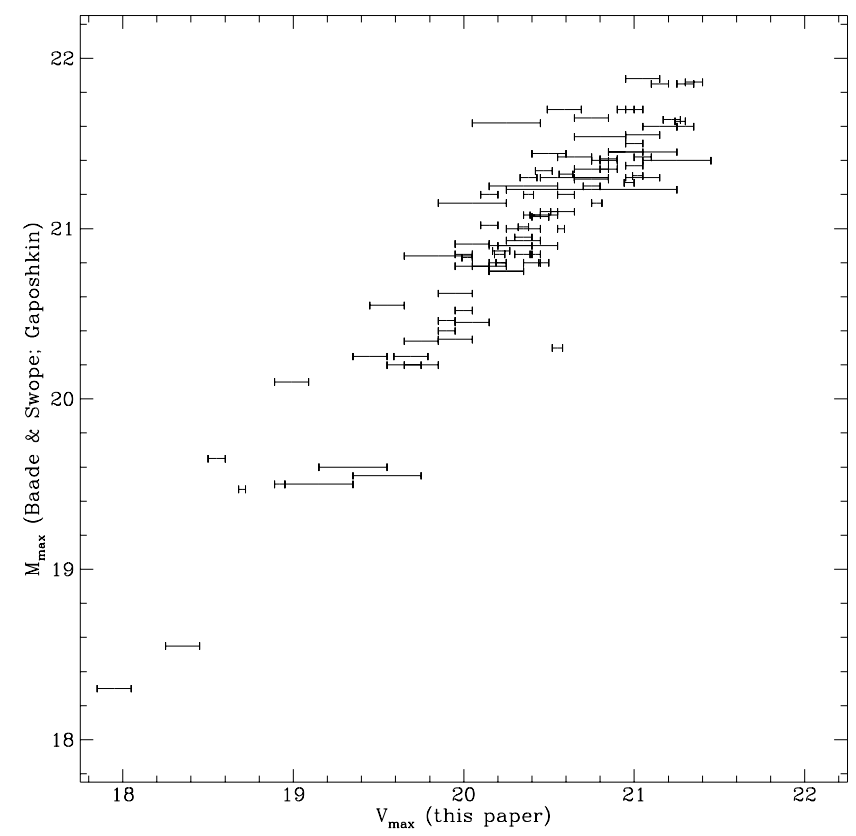

Fig. 3. Comparison of peak magnitudes for M 31 Cepheids observed by us and by Baade \& Swope $(1962,1964)$ or Gaposhkin (1963)

the full period is not covered for many of the variables. Figure 3 shows a comparison of the magnitude at maximum light for the same Cepheids, as determined by us and by Baade \& Swope $(1962,1964)$ and Gaposhkin (1963). The general correlation is clear, though a significant offset can be seen. This offset is due to the fact that the magnitudes reported by Baade and collaborators are photoelectric magnitudes, which are close to $B$, while ours are $V$ magnitudes. The offset of $\sim 0.7$ magnitudes is consistent with the typical colors of these stars.

In Fig. 4 we present a period-luminosity relationship for the Cepheid candidates we have identified, along with those identified by Baade \& Swope $(1962,1964)$ and Gaposhkin (1963). To bring the Baade magnitudes in line with the $V$ magnitudes, we have simply added the 0.7 magnitude offset, which is sufficiently accurate for our purposes here. Included in this diagram is a line representing the position of the PL relationship found by Freedman \& Madore (1990) using improved observations of the Baade \& Swope (1964) Cepheids, from their Field IV, where the extinction is insignificant. It is clear that these variables fall generally along the $P-L$ relationship, lending credence to their identification as Cepheids. One variable which was identified by the light curve turned out to fall far above the $P-L$ relationship and has been rejected as a true Cepheid. This variable is probably of Galactic origin, possibly a binary system in the Galactic halo. There is a small tendency for the points in Fig. 4 to fall below the line for $\log P \sim 1.5$, most likely due to the range of extinction for the sample. For $\log P<1.0$, there is a small

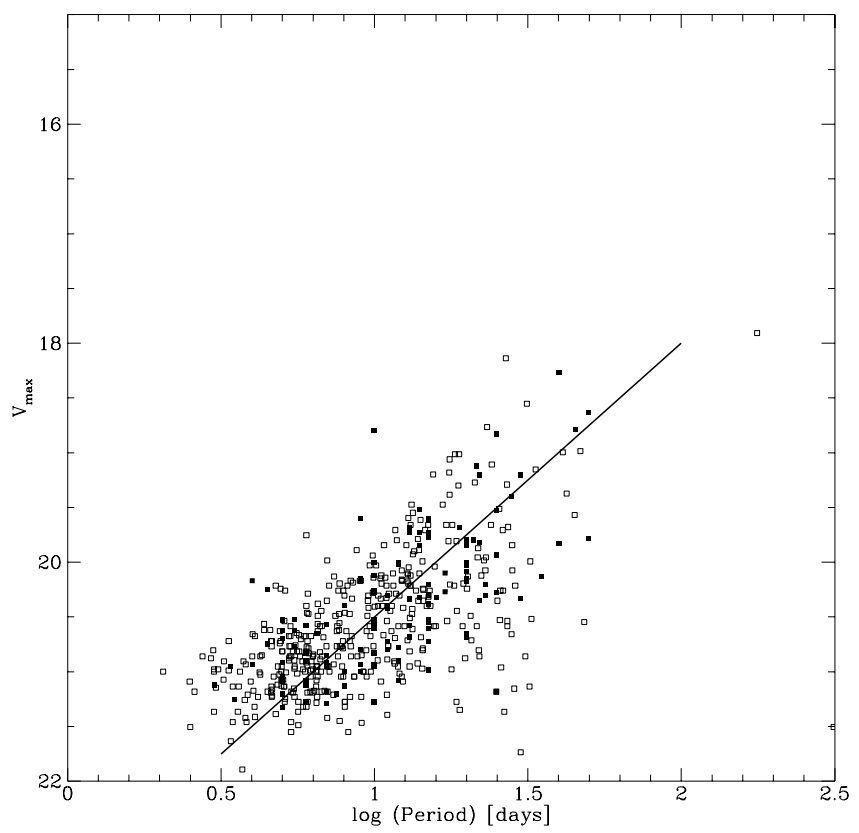

Fig. 4. Period - Luminosity (PL) diagram. The open squares show the measurements of M 31 Cepheids from Baade's group (Baade \& Swope 1962, 1964; Gaposhkin 1963). The filled squares show our new Cepheid candidates. The solid line is the PL relationship determined for a set of Baade M 31 Cepheids by Freedman \& Madore (1990)

tendancy for the points to land above the line. This may be due to Malmquist's bias, to the second track due to Cepheids oscillating at the overtone (e.g., Böhm-Vitense 1994), or because of tendancy for us to underestimate the period (see Fig. 2).

We present lightcurves of all 130 new Cepheid candidates in Figs. 5 - 10 sorted in order of their Right Ascension. Our estimates for the upper and lower limits on $V_{\max }$ are shown in these light curves by two dashed lines. We also tabulate observed quantities of these candidates in Table 1. The candidate rejected on the basis of its location in the $P-L$ diagram is not included in the light curves or table, nor are those Cepheids which were already identified by Baade \& Swope $(1962,1964)$ or Gaposhkin (1963). An estimate of the contamination of our sample can be obtained from the number of nonCepheid variables identified by both Baade's group and our analysis, which we mis-identified as a Cepheid. This number is very low: Of 97 objects which we considered to be Cepheids, none were found to be another kind of variable by Baade's group. This suggests that our contamination is $<1 \%$, but a more conservative estimate might be $3 \%$, because of the low number statistics. The completeness can be estimated in a similar way, by comparing the number of Cepheids which were found in our fields by Baade's group, but which we failed to find. By doing this comparison, we find that our completeness is $53 \%$, while 
the completeness of the Baade dataset is $88 \%$. The magnitude distributions of both samples are similar, and suggest significant lack of completeness due to the magnitude limit for $V_{\max }>21$.

Figure 1 shows the distribution of our newly identified Cepheid variables along with those identified by previous researchers (Baade \& Swope 1962, 1964; Gaposhkin 1963). The reader is cautioned that some of the large-scale structure apparent in this image is due to the limited coverage. The small inset shows the coverage of both our observations and those of Baade \& Swope $(1962,1964)$ and Gaposhkin (1963). We have also included in this figure a contour map from the Hi survey of Unwin (1980). This allows the reader to compare the current location of active star formation with the location of the Cepheids, which have a typical age of roughly $30-125$ Myr. In a companion paper, we will discuss in detail the implications the observed distribution has for the star formation history.

\section{Summary}

We have searched for Cepheid variables in portions of M 31 which have previously been ignored. We have found 130 new Cepheid variable candidates, with $<3 \%$ contamination by other types of variables and a completeness of $\sim 53 \%$ for $V_{\max }<21$. These Cepheids will be used to study the star formation history in M 31.

Acknowledgements. Observations discussed in this paper were performed at the Michigan - Dartmouth - MIT (MDM) Observatory. We would like to thank the MDM TAC for the generous amounts of telescope time devoted to this project. EAM acknowledges support by the Netherlands Foundation for Research in Astronomy (ASTRON) with financial aid from the Netherlands Organization for Scientific Research (NWO) under contract number 782-376-011. Many thanks to Paul Hodge and Yuri Efremov for comments and suggestions.

\section{References}

Baade W., Swope H., 1963, AJ 68, 435

Baade W., Swope H., 1965, AJ 70, 212

Böhm-Vitense E., 1994, AJ 107, 673

Berkhuijsen E.M., Humphreys R.M., Ghigo F.D., Zumach W., 1988, A\&AS 76, 65

Efremov Yu.N., Kopylov I.M., 1967, Izv. Krymsk. Astrofiz. Obs. 36,240

Freedman W., 1989, AJ 98, 1285

Freedman W.L., Madore B.F., 1990, ApJ 365, 186

Gaposchkin S., 1962, AJ 67, 358

Haiman Z., Magnier E.A., Lewin W.H.G., et al., 1993, A\&A 286, 725

Hubble E., 1929, ApJ 69, 103

Magnier E.A., 1996 (in preparation)

Magnier E.A., Lewin W.H.G., van Paradijs J., Hasinger G., Jain A., Pietsch W., Trümper J., 1992, A\&AS 96, 379

Magnier E.A., Lewin W.H.G., van Paradijs J., Hasinger G., Pietsch W., Trümper J., 1993, A\&A 272, 695

Mateo M.L., Schechter P.L., 1989, DoPhot: A CCD Analysis Routine. In: Grosbol P.J., Murtagh F., Warmels R.H. (eds.) 1st ESO/ST - ECF Data Analysis Workshop. European Southern Observatory Garching bei München, p. 213

Metzger M.R., Tonry J.L., Luppino G.A., 1993. In: Hanisch R.J., Brissenden R.J., Barnes J. (eds.) ASP Conf. Ser. 52, 300

Tsvetkov Ts.G., 1988, Ap\&SS 151, 39

Unwin S.C., 1980, MNRAS 192, 243

Welch D.L., McAlary C.W., LcLaren R.A., Madore B.F., 1986, ApJ 305, 583

Young A.T., 1961, PhD Thesis, Harvard University

Young A.T., 1992, in "Automated Telescopes for Photometry and Imaging", ASP Conf. Ser. 28, Adelman A.J., Dukes R.J., Adelman C.J. (eds.) p. 73 